\title{
Modelo Giroscópico para Vibrações em uma Serra de Fita
}

R.D. COPETTI ${ }^{1}$, Departamento de Matemática, UFSM, 97105-900 Santa Maria, RS, Brasil

J.R. CLAEYSSEN ${ }^{2}$, Instituto de Matemática - Promec, UFRGS, 90001-000 Porto Alegre, RS, Brasil.

Resumo. O propósito deste trabalho é determinar a resposta impulso, através da análise modal adjunta de um modelo giroscópico com atrito viscoso para uma serra de fita. O modelo adjunto faz-se necessário devido à natureza não-clássica do problema. A forma dos modos dos sistemas é obtida com o uso da base dinâmica gerada pela resposta impulso e os autovalores, como raízes da equação característica, são obtidos através de uma nova metodologia envolvendo a resposta impulso calculada de maneira não espectral.

\section{Introdução}

A redução de vibração em sistemas dinâmicos giroscópicos em rotação ou translação, por exemplo eixos, serras circulares, correias, serras de fita, e vibração ativa para sistemas, têm recebido crescente atenção devido a suas importantes aplicações em robótica, dinâmica de rotores ou máquinas com alta velocidade de precisão. Nos últimos anos, alguns pesquisadores têm trabalhado nesse tipo de problema ([7], [8]) e, com isso, vários algoritmos para resolvê-los têm sido considerados. Neste trabalho é desenvolvida uma nova metodologia para o cálculo da resposta a um impulso, ou de maneira abreviada, resposta impulso, onde os coeficientes das expansões analíticas da resposta impulso e suas derivadas, que aparecem na equação característica, são gerados de maneira recursiva e correspondem aos valores de uma resposta impulso discreta, os quais dependem dos autovalores. Os primeiros autovalores são calculados como raízes de polinômios que resultam do truncamento das expansões mencionadas, e a forma dos modos dos sistemas é obtida com o uso da base dinâmica gerada pela resposta impulso. Nas simulações numéricas apresentadas verificou-se que o aumento do coeficiente de amortecimento viscoso diminui a amplitude dos modos do sistema giroscópico direto, mas aumenta a amplitude dos modos do sistema giroscópico adjunto, sendo que os autovalores e autofunções ou modos de vibração do problema são complexos. É observado que a resposta impulso, obtida a partir do produto dos modos diretos pelos modos adjuntos, é atenuada com o aumento do coeficiente de atrito viscoso.

\footnotetext{
${ }^{1}$ rmaira@ccne.ufsm.br

${ }^{2}$ julio@mat.ufrgs.br
} 


\section{Equações para o Modelo}

Considera-se um modelo tipo serra de fita controlado através da colocação de dois sensores e atuadores, Fig. 1. Este é um modelo de natureza giroscópica, sujeito a amortecimento viscoso e amortecimento estrutural ou histerético. Como o nosso interesse é o cálculo modal adjunto da resposta impulso, os efeitos do controle serão incluídos dentro de uma força introduzida pelos atuadores.

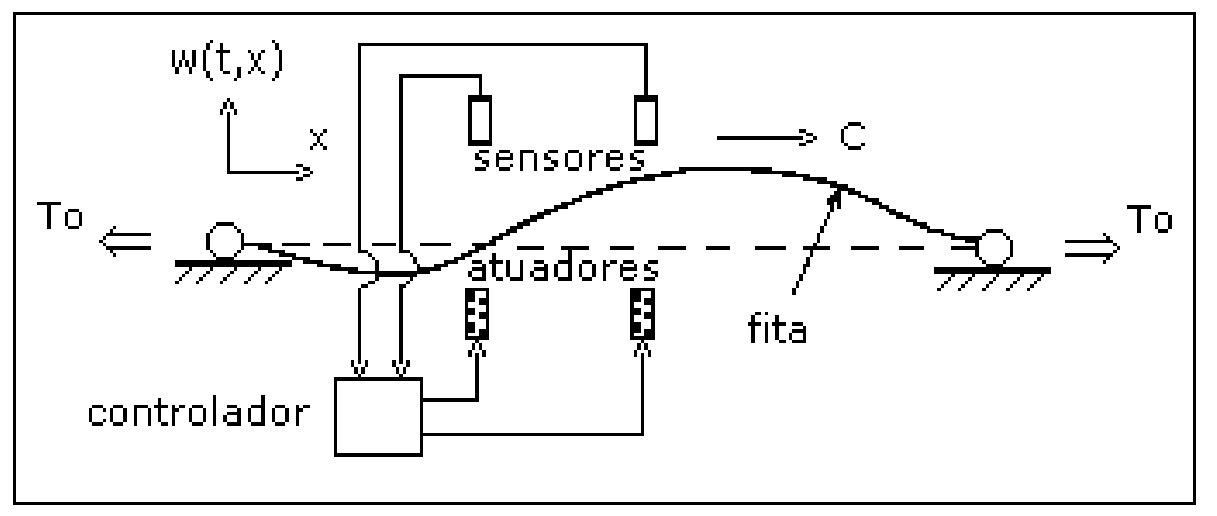

Figura 1: Modelo de uma serra de fita

Assim, o deslocamento transversal $w(t, x)$ é modelado através da equação evolutiva adimensional

$$
\begin{aligned}
& \frac{\partial^{2}}{\partial t^{2}} w(t, x)+\left(2 c \frac{\partial}{\partial x}+d\right) \frac{\partial}{\partial t} w(t, x)+\left(\frac{\partial^{4}}{\partial x^{4}}+\left(k c^{2}-T_{0}\right) \frac{\partial^{2}}{\partial x^{2}}\right) w(t, x) \\
& =f(t, x), \quad x \in(0, L)
\end{aligned}
$$

e condições de contorno

$$
w(t, 0)=0, \quad w(t, L)=0, \quad \frac{\partial^{2}}{\partial x^{2}} w(t, 0)=0, \quad \frac{\partial^{2}}{\partial x^{2}} w(t, L)=0,
$$

onde

$c=$ velocidade de transporte,

$T_{0}=$ tensão na fita,

$d=$ amortecimento viscoso,

$f=$ força de excitação,

$\kappa=$ constante dependente da montagem da polia,

$L=$ comprimento da fita.

\section{Cálculo Modal da Resposta a um Impulso}

A equação (2.1) pode ser escrita na forma matricial evolutiva

$$
\mathcal{M} w_{t t}(t, x)+(\mathcal{D}+\mathcal{G}) w_{t}(t, x)+\mathcal{K} w(t, x)=f(t, x),
$$


com

$\mathcal{M}=I, \quad$ operador identidade,

$\mathcal{D}=d I, \quad \mathrm{~d}$ : coeficiente de amortecimento viscoso,

$\mathcal{G}=2 c \frac{\partial}{\partial x}$, operador espacial de primeira ordem,

c: coeficiente de amortecimento estrutural ou histerético,

$\mathcal{K}=\frac{\partial^{4}}{\partial x^{4}}+\left(\kappa c^{2}-T_{0}\right) \frac{\partial^{2}}{\partial x^{2}}, \quad$ operador espacial de quarta ordem,

sendo que sua solução para condições iniciais $w(0, x)=w_{t}(0, x)=0$ vem a ser a resposta forçada

$$
w(t, x)=\int_{0}^{t} \int_{0}^{L} h(t-\tau, x, \xi) f(\tau, \xi) d \xi d \tau .
$$

Para identificar a resposta impulso $h(t, x, \xi)$, será utilizado o método da variação dos parâmetros. Escrevendo

$$
w(t, x)=\sum_{k=1}^{\infty} c_{k}(t) e^{\lambda_{k} t} u_{k}(x)
$$

substituindo em (3.1) e fazendo a hipótese de Lagrange, tem-se o sistema de equações

$$
\sum_{k=1}^{\infty} e^{\lambda_{k} t} \dot{c}_{k}(t) u_{k}(x)=0 \quad \text { e } \quad \sum_{k=1}^{\infty} \lambda_{k} e^{\lambda_{k} t} \dot{c}_{k}(t) \mathcal{M} u_{k}(x)=f(t, x) .
$$

As funções $c_{k}(t)$ podem ser determinadas a partir da propriedade de ortogonalidade entre as autofunções dos problemas de autovalor de segunda ordem direto e adjunto. Para isto, considera-se o problema de autovalor direto e adjunto, respectivamente,

$$
\left(\lambda_{k}^{2} \mathcal{M}+\lambda_{k} \mathcal{C}+\mathcal{K}\right) u_{k}(x)=0 \quad \text { e } \quad\left(\beta_{j}^{2} \mathcal{M}^{*}+\beta_{j} \mathcal{C}^{*}+\mathcal{K}^{*}\right) v_{j}(x)=0,
$$

onde $\mathcal{C}=\mathcal{D}+\mathcal{G}$. Aqui $\lambda_{k}$ e $u_{k}(x)$ são definidos como sendo o autovalor e a autofunção associados ao problema direto e $\beta_{j}$ e $v_{j}(x)$ são o autovalor e a autofunção associados ao problema adjunto, respectivamente, sendo que os autovalores do problema adjunto podem ser escritos como [4], $\beta_{j}=\bar{\lambda}_{j}, \quad j=1: \infty$. Tomando o produto interno $\langle v, u\rangle=\int_{0}^{L} \bar{v}(x) u(x) d x$, na primeira equação de $(3.5)$ por $v_{j}(x) \mathcal{K}^{*}$, e na segunda por $\bar{\lambda}_{j} v_{j}(x)$, obtém-se:

$$
\sum_{k=1}^{\infty} e^{\lambda_{k} t} \dot{c}_{k}(t)\left\langle v_{j}, \lambda_{k} \lambda_{j} \mathcal{M} u_{k}-\mathcal{K} u_{k}\right\rangle=\left\langle v_{j}, \lambda_{j} f\right\rangle, \quad j=1: \infty .
$$

Com uma conveniente ordenação dos autovalores e das autofunções do problema direto e do correspondente problema adjunto, é possível assumirmos que as autofunções satisfazem à relação de ortogonalidade (a prova deste fato pode ser encontrada em [4] )

$$
\left\langle v_{j}, \lambda_{j} \lambda_{k} \mathcal{M} u_{j}-\mathcal{K} u_{j}\right\rangle=0, j \neq k \quad \text { e } \quad \gamma_{k}=\left\langle v_{k}, \lambda_{k}^{2} \mathcal{M} u_{k}-\mathcal{K} u_{k}\right\rangle \neq 0, \quad j=k
$$


decorre então, de (3.7), que $\dot{c}_{k}(t)=\frac{\lambda_{k}}{\gamma_{k}} e^{-\lambda_{k} t}\left\langle v_{k}, f\right\rangle$. Integrando de 0 a $t$ ambos os lados da equação anterior, segue

$$
c_{k}(t)=\frac{\lambda_{k}}{\gamma_{k}} \int_{0}^{t} e^{-\lambda_{k} \tau}\left\langle v_{k}(x), f(\tau, x)\right\rangle d \tau,
$$

onde a constante $c_{k}(0)$ é igual a zero pelas condições iniciais nulas do problema (3.1).

Substituindo (3.9) em (3.4), tem-se

$$
u(t, x)=\int_{0}^{t} \int_{0}^{L}\left(\sum_{k=1}^{\infty} \frac{\lambda_{k}}{\gamma_{k}} e^{\lambda_{k}(t-\tau)} u_{k}(x) \bar{v}_{k}(\xi)\right) f(\tau, \xi) d \xi d \tau,
$$

de modo que a resposta impulso pode ser identificada através de (3.3) como sendo

$$
h(t, x, \xi)=\sum_{k=1}^{\infty} \frac{\lambda_{k}}{\gamma_{k}} e^{\lambda_{k} t} u_{k}(x) \bar{v}_{k}(\xi),
$$

onde $\gamma_{k}$ é a constante definida por (3.8), $u_{k}(x)$ são as autofunções associadas aos autovalores $\lambda_{k}$ do problema de segunda ordem direto e $v_{k}(x)$ são as autofunções do problema adjunto, obtidos a seguir.

\subsection{Cálculo dos Modos da Equação Evolutiva Direta}

A busca de soluções do tipo $w(t, x)=e^{\lambda t} \phi(x)$ para o problema homogêneo associado a $(3.1)$,

$$
\left(\mathcal{M} \frac{\partial^{2}}{\partial t^{2}}+(\mathcal{D}+\mathcal{G}) \frac{\partial}{\partial t}+\mathcal{K}\right) w(t, x)=0,
$$

onde os operadores foram definidos em (3.2), leva à resolução do problema de autovalor

$$
\frac{\partial^{4}}{\partial x^{4}} \phi(x)+\left(\kappa c^{2}-T_{0}\right) \frac{\partial^{2}}{\partial x^{2}} \phi(x)+2 \lambda c \frac{\partial}{\partial x} \phi(x)+\left(\lambda d+\lambda^{2}\right) \phi(x)=0,
$$

onde $\lambda$ é o autovalor associado à autofunção $\phi(x)$, que deve satisfazer as condições de contorno

$$
\phi(0)=\phi(L)=\phi^{\prime \prime}(0)=\phi^{\prime \prime}(L)=0 .
$$

A solução geral da equação acima é da forma

$$
\phi(x)=c_{1} \phi_{1}(x)+c_{2} \phi_{2}(x)+c_{3} \phi_{3}(x)+c_{4} \phi_{4}(x),
$$

ou, na forma matricial, $\phi(x)=\widehat{\Phi} C$, onde $\widehat{\Phi}=\left[\begin{array}{lll}\phi_{1} & \phi_{2} & \phi_{3}\end{array} \phi_{4}\right]$ é uma base de soluções e $C=\left[\begin{array}{llll}c_{1} & c_{2} & c_{3} & c_{4}\end{array}\right]^{T}$ um vetor de componentes constantes. Em termos dessa base, o problema de contorno pode ser escrito

$$
\mathrm{U} C=0, \quad \mathrm{U}=B \Phi,
$$


sendo

$$
B=\left[\begin{array}{llllllll}
1 & 0 & 0 & 0 & 0 & 0 & 0 & 0 \\
0 & 0 & 1 & 0 & 0 & 0 & 0 & 0 \\
0 & 0 & 0 & 0 & 1 & 0 & 0 & 0 \\
0 & 0 & 0 & 0 & 0 & 0 & 1 & 0
\end{array}\right], \Phi=\left[\begin{array}{cccc}
\phi_{1}(0) & \phi_{2}(0) & \phi_{3}(0) & \phi_{4}(0) \\
\phi_{1}^{\prime}(0) & \phi_{2}^{\prime}(0) & \phi_{3}^{\prime}(0) & \phi_{4}^{\prime}(0) \\
\phi_{1}^{\prime \prime}(0) & \phi_{2}^{\prime \prime}(0) & \phi_{3}^{\prime \prime}(0) & \phi_{4}^{\prime \prime}(0) \\
\phi_{1}^{\prime \prime \prime}(0) & \phi_{2}^{\prime \prime \prime}(0) & \phi_{3}^{\prime \prime}(0) & \phi_{4}^{\prime \prime \prime}(0) \\
\phi_{1}(L) & \phi_{2}(L) & \phi_{3}(L) & \phi_{4}(L) \\
\phi_{1}^{\prime}(L) & \phi_{2}^{\prime}(L) & \phi_{3}^{\prime}(L) & \phi_{4}^{\prime}(L) \\
\phi_{1}^{\prime \prime}(L) & \phi_{2}^{\prime \prime}(L) & \phi_{3}^{\prime \prime}(L) & \phi_{4}^{\prime \prime}(L) \\
\phi_{1}^{\prime \prime \prime}(L) & \phi_{2}^{\prime \prime \prime}(L) & \phi_{3}^{\prime \prime \prime}(L) & \phi_{4}^{\prime \prime \prime}(L)
\end{array}\right]
$$

Os autovalores em (3.11) podem ser determinados escolhendo-se como base, $\widehat{\Phi}$, a base dinâmica, isto é, tomando

$$
\phi_{1}(x)=h(x), \quad \phi_{2}(x)=h^{\prime}(x), \quad \phi_{3}(x)=h^{\prime \prime}(x), \quad \phi_{4}(x)=h^{\prime \prime \prime}(x),
$$

onde $h(x)$ é a solução fundamental descrita como solução do problema de valor inicial

$$
\begin{aligned}
& \frac{\partial^{4}}{\partial x^{4}} h(x)+\left(\kappa c^{2}-T_{0}\right) \frac{\partial^{2}}{\partial x^{2}} h(x)+2 \lambda c \frac{\partial}{\partial x} h(x)+\left(\lambda d+\lambda^{2}\right) h(x)=0, \\
& h(0)=h^{\prime}(0)=h^{\prime \prime}(0)=0, \quad h^{\prime \prime \prime}(0)=1 .
\end{aligned}
$$

Com a escolha da base dinâmica, tem-se que os autovalores são raízes da equação característica

$$
\operatorname{det}(\mathbf{U})=\phi_{1}(L) \phi_{3}^{\prime \prime}(L)-\phi_{3}(L) \phi_{1}^{\prime \prime}(L)=0 .
$$

A forma analítica (shape) dos modos diretos $u$ pode ser obtida com a resolução do problema (3.12), sendo $\widehat{\Phi}$ a base dada em (3.13). Para tanto, serão utilizadas as condições iniciais de $h(x)$ em $x=0$. A escolha da base dinâmica implica que $c_{2}=c_{4}=0$ e o sistema (3.12) é reduzido a

$$
\left[\begin{array}{cc}
h(L) & h^{\prime \prime}(L) \\
h^{\prime \prime}(L) & h^{\prime}(L)
\end{array}\right]\left[\begin{array}{l}
c_{1} \\
c_{3}
\end{array}\right]=\left[\begin{array}{l}
0 \\
0
\end{array}\right] .
$$

Decorre que o modo $u$, correspondente ao autovalor $\lambda$, é dado por

$$
u=\sigma(\lambda) h(x, \lambda)+h^{\prime \prime}(x, \lambda),
$$

onde $h(x, \lambda)$ é a solução de (3.14) e $\sigma$ é o fator $\sigma=-\frac{h^{\prime \prime}(L, \lambda)}{h(L, \lambda)}$.

\subsection{Cálculo dos Modos da Equação Evolutiva Adjunta}

Para determinar $v_{k}(x)$, em (3.6), torna-se necessário considerar a equação diferencial adjunta associada ao problema homogêneo (3.1) dada por

$$
\left(\mathcal{M}^{*} \frac{\partial^{2}}{\partial t^{2}}-\left(\mathcal{D}^{*}+\mathcal{G}^{*}\right) \frac{\partial}{\partial t}+\mathcal{K}^{*}\right) \mathrm{w}(t, x)=0,
$$


onde $\left(^{*}\right)$ denota o operador adjunto associado aos operadores espaciais $\mathcal{M}, \mathcal{C}=$ $\mathcal{D}+\mathcal{G}$ e $\mathcal{K}$, dados por

$$
\begin{array}{ll}
\mathcal{M}^{*}=I, & \mathcal{D}^{*}=d I, \\
\mathcal{G}^{*}=-2 c \frac{\partial}{\partial x}, & \mathcal{K}^{*}=\frac{\partial^{4}}{\partial x^{4}}+\left(k c^{2}-T_{0}\right) \frac{\partial^{2}}{\partial x^{2}} .
\end{array}
$$

Para o problema da fita modelado por uma viga biapoiada, as condições de contorno do problema adjunto são as mesmas que as do problema direto. Assim, a equação adjunta evolutiva é

$$
\frac{\partial^{2}}{\partial t^{2}} \mathbf{w}(t, x)+\left(2 c \frac{\partial}{\partial x}-d\right) \frac{\partial}{\partial t} \mathbf{w}(t, x)+\left(\frac{\partial^{4}}{\partial x^{4}}+\left(k c^{2}-T_{0}\right) \frac{\partial^{2}}{\partial x^{2}}\right) \mathbf{w}(t, x)=f(t, x),
$$

cujas condições de contorno adjuntas são

$$
\mathrm{w}(t, 0)=0, \quad \mathrm{w}(t, L)=0, \quad \frac{\partial^{2}}{\partial x^{2}} \mathrm{w}(t, 0)=0, \quad \frac{\partial^{2}}{\partial x^{2}} \mathrm{w}(t, L)=0 .
$$

Através de um raciocínio análogo ao do problema direto e pelas condições de contorno autoadjuntas, obtém-se que a forma analítica dos modos adjuntos $v$ é a mesma do problema direto, isto é, considerando, $\mathrm{w}(t, x)=c_{k} e^{\beta_{k} t} v_{k}(x)$, tem-se

$$
v=\sigma(\lambda) h(x, \beta)+h^{\prime \prime}(x, \beta),
$$

onde $\sigma$ é o fator

$$
\sigma=-\frac{h^{\prime \prime}(L, \beta)}{h(L, \beta)}
$$

$\beta$ é autovalor do problema espacial adjunto

$$
\begin{aligned}
& \frac{\partial^{4}}{\partial x^{4}} \psi(x)+\left(\kappa c^{2}-T_{0}\right) \frac{\partial^{2}}{\partial x^{2}} \psi(x)-2 \beta c \frac{\partial}{\partial x} \psi(x)+\left(\beta^{2}+\beta d\right) \psi(x)=0, \\
& \psi(0)=\psi(L)=\psi^{\prime \prime}(0)=\psi^{\prime \prime}(L)=0,
\end{aligned}
$$

e $h(x, \beta)$ é a solução do problema de valor inicial adjunto

$$
\begin{aligned}
& \frac{\partial^{4}}{\partial x^{4}} h(x)+\left(\kappa c^{2}-T_{0}\right) \frac{\partial^{2}}{\partial x^{2}} h(x)-2 \beta c \frac{\partial}{\partial x} h(x)+\left(\beta^{2}+\beta d\right) h(x)=0, \\
& h(0)=h^{\prime}(0)=h^{\prime \prime}(0)=0, \quad h^{\prime \prime \prime}(0)=1 .
\end{aligned}
$$

\subsection{Cálculo dos Autovalores através da Resposta a um Im- pulso Discreta}

A determinação dos autovalores do problema direto, $\lambda$, e adjunto, $\beta$, envolve a resolução dos problemas espaciais de autovalor direto e adjunto, respectivamente, onde para este cálculo, a solução fundamental, h(x), pode ser utilizada. Essa solução pode ser obtida na forma espectral para o caso de raízes distintas

$$
h(x)=\sum_{j=1}^{4} \frac{e^{\rho_{j} x}}{P^{\prime}\left(\rho_{j}\right)},
$$


onde os $\rho_{j}$ são as raízes do polinômio característico

$$
P(s)=s^{4}+\left(\kappa c^{2}-T_{0}\right) s^{2}+2 \lambda_{k} c s+\lambda_{k}^{2}+d \lambda_{k}
$$

do problema direto (3.11), ou

$$
P(s)=s^{4}+\left(\kappa c^{2}-T_{0}\right) s^{2}-2 \beta c s+\beta^{2}+d \beta
$$

para o problema adjunto (3.15).

Numa forma não espectral, a solução fundamental $h(x)$ pode ser determinada através de uma inversão da transformada de Laplace de $\frac{1}{P(s)}$ ou, como uma série de potências

$$
h(x)=\sum_{k=0}^{\infty} \frac{x^{k}}{k !} h_{k}, \quad h_{k}=h^{k}(0)
$$

onde $h_{k}$ vem a ser a solução discreta fundamental ou resposta impulso discreta da equação em diferenças associada com o problema direto, isto é,

$$
\begin{aligned}
& h_{k+4}+\left(\kappa c^{2}-T_{0}\right) h_{k+2}+2 \lambda c h_{k+1}+\left(\lambda d+\lambda^{2}\right) h_{k}=0, \\
& h_{0}=h_{1}=h_{2}=0 \quad h_{3}=1 .
\end{aligned}
$$

Para o problema adjunto, tem-se a seguinte equação para a correspondente resposta impulso discreta:

$$
\begin{aligned}
& h_{k+4}+\left(\kappa c^{2}-T_{0}\right) h_{k+2}-2 \beta c h_{k+1}+\left(\beta^{2}+\beta d\right) h_{k}=0, \\
& h_{0}=h_{1}=h_{2}=0 \quad h_{3}=1 .
\end{aligned}
$$

Uma maneira de se obter os autovalores aproximados consiste no uso da forma não espectral para $\mathrm{h}(\mathrm{x})$ e suas derivadas em séries de potências e truncá-las com $\mathrm{N}$ termos. Os valores de $h_{k}$ são obtidos de forma recursiva.

\section{Simulações Numéricas}

Foram realizadas várias simulações numéricas para diversos valores do atrito estrutural ou histerético $d$. Os parâmetros usados nestas simulações são dados na Tabela 1 ([7]). Para cinco desses valores, e com uma série de $N=100$ termos, foram obtidos os autovalores listados na Tabela 2. A esta tabela adicionam-se os correspondentes autovalores complexos conjugados.

\subsection{Caso Giroscópico Não-Amortecido}

No caso giroscópico não-amortecido $\mathcal{D}=0(\mathrm{~d}=0)$, o problema é autoadjunto, de modo que os modos do problema adjunto são os mesmos do problema direto e a resposta impulso dada em (3.10) envolve somente os autovalores e autovetores do problema direto. Na Figura 2, encontram-se simulações do primeiro, terceiro, 


\begin{tabular}{cc}
\hline \hline Parâmetro & Valor numérico \\
\hline \hline$c$ & 10 \\
$T_{0}$ & 100 \\
$L$ & 1 \\
$\kappa$ & 0.5 \\
\hline
\end{tabular}

Tabela 1: Valores dos parâmetros para o modelo da serra de fita

\begin{tabular}{ccccc}
\hline \hline $\mathrm{d}=-10$ & $\mathrm{~d}=-5$ & $\mathrm{~d}=0$ & $\mathrm{~d}=5$ & $\mathrm{~d}=10$ \\
\hline \hline $2.577+17.164 \mathrm{i}$ & $1.289+17.315 \mathrm{i}$ & $17.365 \mathrm{i}$ & $-1.289+17.315 \mathrm{i}$ & $-2.577+17.164 \mathrm{i}$ \\
$3.956+53.935 \mathrm{i}$ & $1.977+54.034 \mathrm{i}$ & $54.066 \mathrm{i}$ & $-1.977+54.034 \mathrm{i}$ & $-3.956+53.935 \mathrm{i}$ \\
$4.778+111.595 \mathrm{i}$ & $2.388+111.661 \mathrm{i}$ & $111.682 \mathrm{i}$ & $-2.388+111.661 \mathrm{i}$ & $-4.778+111.595 \mathrm{i}$ \\
$5.140+187.565 \mathrm{i}$ & $2.569+187.612 \mathrm{i}$ & $187.627 \mathrm{i}$ & $-2.569+187.612 \mathrm{i}$ & $-5.140+187.565 \mathrm{i}$ \\
$5.261+281.298 \mathrm{i}$ & $2.630+281.332 \mathrm{i}$ & $281.343 \mathrm{i}$ & $-2.630+281.332 \mathrm{i}$ & $-5.261+281.298 \mathrm{i}$ \\
$5.279+393.237 \mathrm{i}$ & $2.639+393.262 \mathrm{i}$ & $393.270 \mathrm{i}$ & $-2.639+393.262 \mathrm{i}$ & $-5.279+393.237 \mathrm{i}$ \\
\hline
\end{tabular}

Tabela 2: Autovalores para o modelo da serra de fita

quarto e sexto modos para o modelo da serra de fita, no caso não-amortecido. $\mathrm{Na}$ legenda, (Re) indica a parte real do modo e $(\mathrm{Im})$ a respectiva parte imaginária.

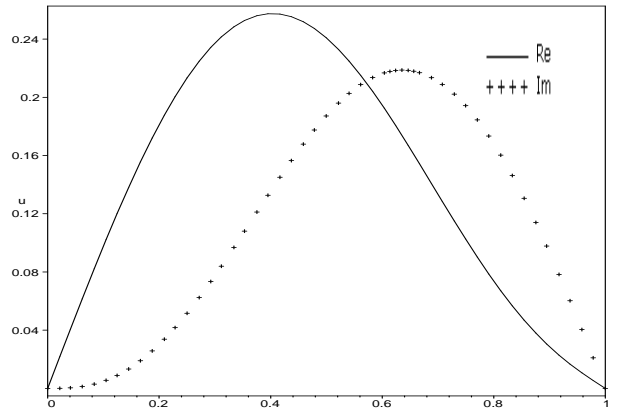

(a) primeiro modo

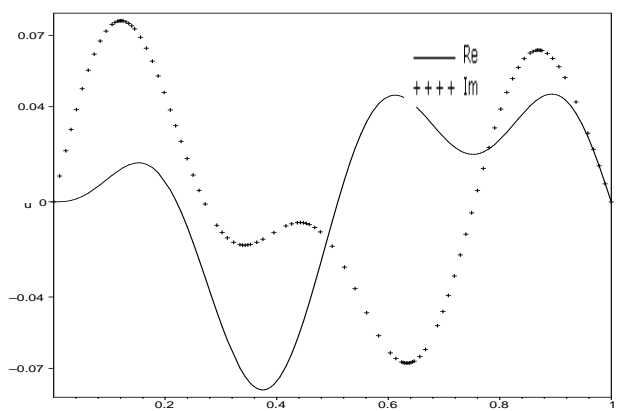

(c) quarto modo

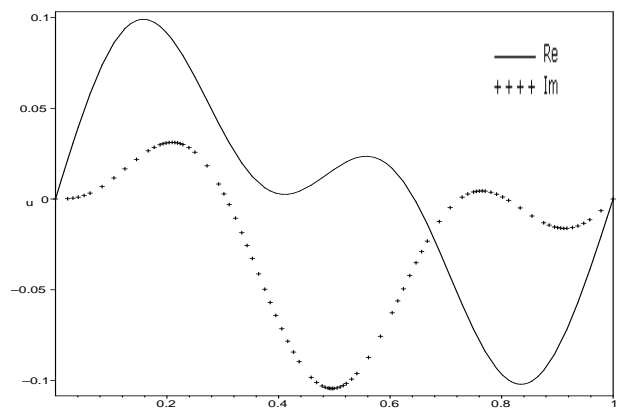

(b) terceiro modo

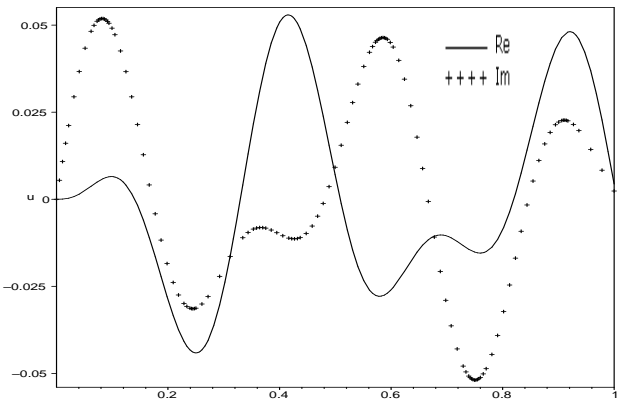

(d) sexto modo

Figura 2: Modos para o sistema giroscópico não-amortecido 


\subsection{Caso Giroscópico Amortecido}

Para o caso em que $\mathcal{D} \neq 0$, tem-se que a resposta impulso envolve o cálculo das autofunções ou modos associadas aos autovalores $\lambda_{k}$ do problema direto, $u_{k}(x)$, e das autofunções ou modos do problema adjunto, $v_{k}(x)$. Nas Figuras 3 e 4 têm-se simulações para alguns modos do sistema direto para três valores do parâmetro $d$, $(d=-10, d=0, d=10)$, observando-se que a amplitude dos mesmos aumenta conforme $d$ diminui, porém mantendo-se a forma dos modos, conforme esperado. Nas simulações realizadas para os correspondentes modos adjuntos, observou-se que a amplitude dos modos adjuntos aumenta conforme $d$ aumenta, na mesma razão que a amplitude dos modos diretos diminui.

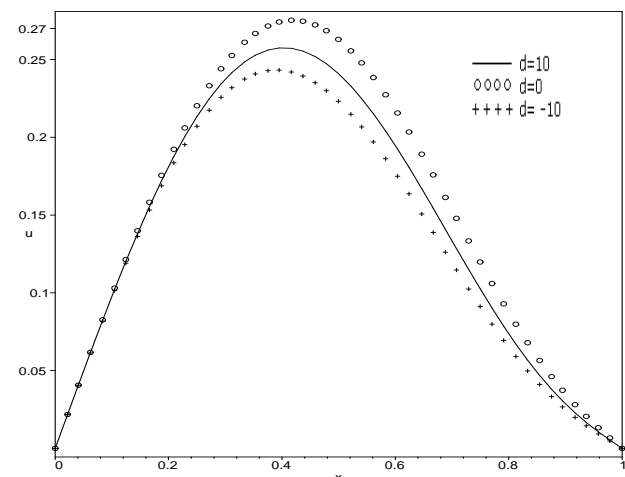

(a) parte real

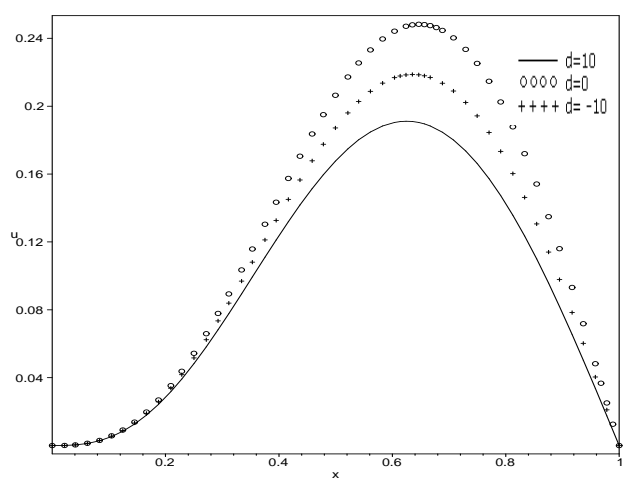

(b) parte imaginária

Figura 3: Primeiro modo do sistema giroscópico direto

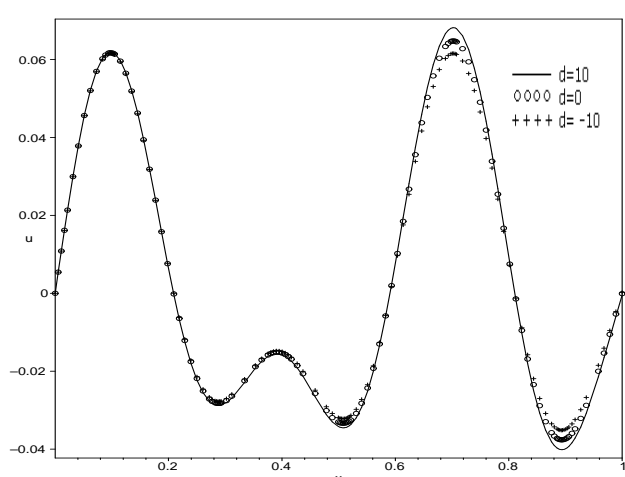

(a) parte real

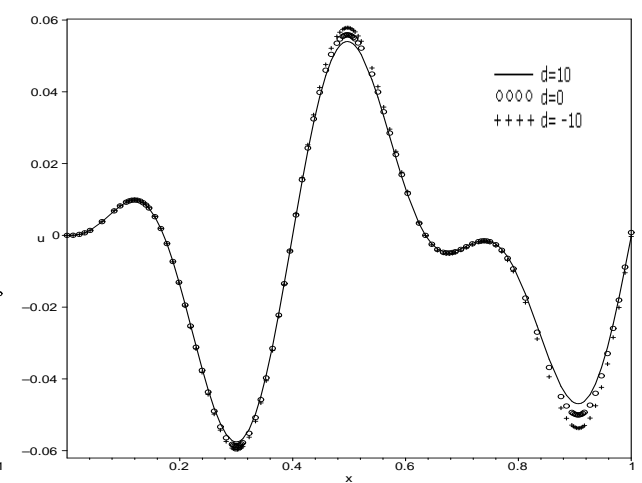

(b) parte imaginária

Figura 4: Quinto modo do sistema giroscópico direto 


\section{Conclusão}

A resposta impulso de um modelo não-clássico foi obtida através do método modal adjunto. Este método foi aplicado diretamente no espaço físico do problema, sem o uso da formulação de espaço de estado, comumente usada na literatura. Foram resolvidos o problema de autovalor direto e o problema adjunto de autovalor. Este método mostrou-se eficiente uma vez que permitiu escrever de maneira espectral a resposta impulso em função dos autovalores e autofunções do sistema direto e adjunto. Foram realizadas várias simulações numéricas, para diversos valores do amortecimento viscoso, onde observou-se que, tanto para o sistema direto quanto para o sistema adjunto, os modos obtidos desta maneira mantiveram a forma esperada.

Abstract. The purpose of this work is to determine the impulse response through adjoint modal analysis, of a gyroscopic viscous damped model for a band saw. The adjoint model turns out to be necessary due to no-classic nature of the problem. The mode shapes of the systems are obtained with the use of the dynamical basis generated by the impulse response and the eigenvalues, as roots of the characteristic equation, are obtained through a new methodology involving the impulse response calculated in a non-spectral form.

\section{Referências}

[1] A.G. Butkovskiy, "Structural Theory of Distributed Systems", John Wiley, New York, 1983.

[2] J.R. Claeyssen, L.D. Chiwiacowsky e G.C. Suazo, The impulse response in the symbolic computating of modes for beams and plates, Applied Numerical Mathematics, 40 (2002), 119-135.

[3] R.D. Copetti e J.R. Claeyssen, Vibrações forçadas em uma viga fixa-livre sujeita a um torque e movimento no apoio, em "Seleta do XXIV CNMAC" (J.M. Balthazar, S. M. Gomes e A. Sri Ranga, eds), Tend. Mat. Apl. Comput., 3, No. 1 (2002), 81-90.

[4] R.D. Copetti, "Sistemas Concentrados e Distribuídos através da Análise Modal Adjunta", Tese de Doutorado, PROMEC, UFRGS, Porto Alegre, RS, 2002.

[5] J.H. Ginsberg, "Mechanical and Structural Vibrations - Theory and Applications", John Wiley, New York, 2001.

[6] V. Jr. Steffen, Vibrações laterais de um fio auto-excitado por seu movimento axial, em "Anais do VI Congresso Brasileiro de Engenharia Mecânica", C-2, pp. 11-19, 1981.

[7] B. Yang, Vibration control of gyroscopic systems via direct velocity feedback, Journal of Sound and Vibration, 175, No. 4 (1994), 525-534.

[8] J.A. Wickert e C.D. Jr. Mote, Classical vibration analysis of axially moving continua, Journal of Applied Mechanics, 57 (1990), 738-744. 